\title{
Generating function for $K$-restricted jagged partitions
}

\author{
J.-F. Fortin*, P. Jacob`and P. Mathieu \\ Département de physique, de génie physique et d'optique, \\ Université Laval, \\ Québec, Canada, G1K 7P4.
}

Submitted: Oct 9, 2004; Accepted: Feb 11, 2005; Published: Feb 21, 2005

Mathematics Subject Classifications: 05A15, 05A17, 05A19

\begin{abstract}
We present a natural extension of Andrews' multiple sums counting partitions of the form $\left(\lambda_{1}, \cdots, \lambda_{m}\right)$ with $\lambda_{i} \geq \lambda_{i+k-1}+2$. The multiple sum that we construct is the generating function for the so-called $K$-restricted jagged partitions. A jagged partition is a sequence of non-negative integers $\left(n_{1}, n_{2}, \cdots, n_{m}\right)$ with $n_{m} \geq 1$ subject to the weakly decreasing conditions $n_{i} \geq n_{i+1}-1$ and $n_{i} \geq n_{i+2}$. The $K$-restriction refers to the following additional conditions: $n_{i} \geq n_{i+K-1}+1$ or $n_{i}=n_{i+1}-1=$ $n_{i+K-2}+1=n_{i+K-1}$. The corresponding generalization of the Rogers-Ramunjan identities is displayed, together with a novel combinatorial interpretation.
\end{abstract}

\section{Introduction}

In 1981 Andrews [2] showed that the generating function for partitions with prescribed number of parts subject to the following difference 2 condition

$$
\lambda_{j} \geq \lambda_{j+k-1}+2
$$

and containing at most $i-1$ parts equal to 1 is

$$
F_{k, i}(z ; q)=\sum_{m_{1}, \cdots, m_{k-1}=0}^{\infty} \frac{q^{N_{1}^{2}+\cdots+N_{k-1}^{2}+L_{i}} z^{N}}{(q)_{m_{1}} \cdots(q)_{m_{k-1}}},
$$

*Present address: Department of Physics and Astronomy, Rutgers, the State University of New Jersey, Piscataway, NJ 08854-8019; jffor27@physics.rutgers.edu

${ }^{\dagger}$ Present address: Department of Mathematical Sciences, University of Durham, Durham, DH1 3L, UK; patrick.jacob@durham.ac.uk

${ }^{\ddagger}$ pmathieu@phy.ulaval.ca. This work is supported by NSERC. 
with

$$
N_{j}=m_{j}+\cdots+m_{k-1}, \quad L_{j}=N_{j}+\cdots N_{k-1}, \quad N=L_{1},
$$

$\left(L_{k}=L_{k+1}=0\right)$ and

$$
(a)_{n}=(a ; q)_{n}=\prod_{i=0}^{n-1}\left(1-a q^{i}\right) .
$$

This is a one-parameter deformation of the multiple $q$-series of the analytic AndrewsGordon identity [1, 3].

In this work, we present the derivation of the generating function for jagged partitions of length $m$, which are sequences of non-negative integers $\left(n_{1}, n_{2}, \cdots, n_{m}\right)$ satisfying

$$
n_{j} \geq n_{j+1}-1, \quad n_{j} \geq n_{j+2}, \quad n_{m} \geq 1,
$$

and further subject to the following $K$-restrictions:

$$
n_{j} \geq n_{j+K-1}+1 \quad \text { or } \quad n_{j}=n_{j+1}-1=n_{j+K-2}+1=n_{j+K-1},
$$

for all values of $j \leq m-K+1$, with $K>2$. Following [2], the derivation of the generating function uses a recurrence process controlled by a boundary condition. In the present case, the boundary condition is a constraint on the number of pairs 01 that can appear in the $K$-restricted jagged partitions. Our main result is the following (which is a reformulation of Theorem 7, section 3):

Theorem 1. If $A_{K, 2 i}(m, n)$ stands for the set of non-negative integer sequences $\left(n_{1}, \cdots, n_{m}\right)$ of weight $n=\sum_{j=1}^{m} n_{j}$ satisfying the weakly decreasing conditions (5) together with the restrictions (6) and containing at most $i-1$ pairs 01 , then its generating function is

$$
\sum_{n, m \geq 0} A_{K, 2 i}(m, n) z^{m} q^{n}=\sum_{m_{0}, \cdots, m_{\kappa-1}=0}^{\infty} \frac{q^{m_{0}\left(m_{0}+1\right) / 2+\epsilon m_{0} m_{\kappa-1}+N_{1}^{2}+\cdots+N_{\kappa-1}^{2}+L_{i}} z^{m_{0}+2 N}}{(q)_{m_{0}} \cdots(q)_{m_{\kappa-1}}}
$$

where $\kappa$ and $\epsilon(=0$ or 1$)$ are related to $K$ by $K=2 \kappa-\epsilon$ and where $N_{j}$ and $L_{j}$ are given in (3) with $k$ replaced by $\kappa$.

Jagged partitions have first been introduced in the context of a conformal-field theoretical problem [15]. In that framework, $K=2 \kappa$, i.e., it is an even integer. The generating function for the $2 \kappa$-restricted jagged partitions with boundary condition specified by $i$ has been found in [5]. It is related to the character of the irreducible module of the parafermionic highest-weight state specified by a singular-vector condition labeled by the integer $1 \leq i \leq \kappa$.

Our essential contribution in this paper is to present the generating function for $K$ odd. This is certainly a very natural extension to consider and it turns out to be not so straightforward. Moreover, the resulting generating function has a nontrivial product form, which is given in Theorem 11 (in the even case, the product form reduces to the usual one in the Andrews-Gordon identity [1]). In all but one case, the resulting generalizations of Rogers-Ramanujan identities reduce to identities already found by Bressoud 
[6]. However, the identity corresponding to $i=\kappa$ (with $K=2 \kappa-1$ ) appears to be new. But quite interestingly, in all cases (i.e., for all allowed values of $i$ and $K$, including $K$ even), we present (in Corollary 12) a new combinatorial interpretation of these generalized Rogers-Ramanujan identities in terms of jagged partitions. The significance of this work lies more in this new interpretation of these identities than in the novelty of the results.

Somewhat unexpectingly, a physical realization of the $K$-restricted jagged partitions for $K$ odd has been found recently, in the context of superconformal minimal models [14].

\section{Jagged partitions}

Let us start by formalizing and exemplifying the notions of jagged partitions and their restrictions.

Definition 2. A jagged partition of length $m$ is a sequence of $m$ non-negative integers $\left(n_{1}, n_{2}, \cdots, n_{m}\right)$ satisfying $n_{j} \geq n_{j+1}-1, n_{j} \geq n_{j+2}$ and $n_{m} \geq 1$.

Notice that even if the last entry is strictly positive, some zero entries are allowed. For instance, the lowest-weight jagged partition is of the form $(\cdots 01010101)$. The origin of the qualitative 'jagged' is rooted in the jagged nature of this lowest-weight sequence. The list of all jagged partitions of length 6 and weight 7 is:

$\{(410101),(320101),(230101),(311101),(221101),(212101),(211111),(121111),(121201)\}$.

Observe that to the set of integers $\{0,1,1,1,2,2\}$ there correspond three jagged partitions of length 6 and weight 7 but, of course, only one standard partition.

Definition 3. A $K$-restricted jagged partition of length $m$ is a jagged partition further subject to the conditions: $n_{j} \geq n_{j+K-1}+1$ or $n_{j}=n_{j+1}-1=n_{j+K-2}+1=n_{j+K-1}$ (called $K$-restrictions) for all values of $j \leq m-K+1$, with $K>2$.

The first condition enforces a difference-one condition between parts separated by a distance $K-1$ in the sequence. However, the second condition allows for some partitions with difference 0 between parts at distance $K-1$ if in addition they satisfy an in-between difference 2 at distance $K-3$. In other words, it is equivalent to $n_{j}=n_{j+K-1}$ and $n_{j+1}=n_{j+K-2}+2$. The general pattern of such $K$ consecutive numbers is $(n, n+$ $1, \cdots, n-1, n)$, where the dots stand for a sequence of $K-4$ integers compatible with the weakly decreasing conditions (5).

The list of all 5-restricted jagged partitions of length 6 and weight 7 is

$$
\{(320101),(230101),(221101),(212101),(121201)\} \text {. }
$$

Comparing this list with that in (8), we see that (410101) is not allowed since $n_{2}=n_{6}$ but $n_{3} \neq n_{5}+2$. (311101) and (211111) are excluded for the same reason. Moreover, (1211101) is excluded since $n_{1}=n_{5}$ but $n_{2} \neq n_{4}+2$. (212101) is an example of an 
allowed jagged partition with an in-between difference 2 condition for parts separated by the distance $K-3=2$.

\section{Recurrence relations for generating functions}

We first introduce two sets of $K$-restricted jagged partitions with prescribed boundary conditions:

$A_{K, 2 i}(m, n)$ : the number of $K$-restricted jagged partitions of $n$ into $m$ parts with at most $(i-1)$ pairs of 01 , with $1 \leq i \leq[(K+1) / 2]$.

$B_{K, j}(m, n)$ : the number of $K$-restricted jagged partitions of $n$ into $m$ parts with at most $(j-1)$ consecutive 1 's at the right end, with $1 \leq j \leq K$.

These definitions are augmented by the specification of the following boundary conditions:

$$
A_{K, 2 i}(0,0)=B_{K, j}(0,0)=1, \quad A_{K, 0}(m, n)=B_{K, 0}(m, n)=0 .
$$

Moreover, it will be understood that both $A_{K, 2 i}(m, n)$ and $B_{K, j}(m, n)$ are zero when either $m$ or $n$ is negative and if either of $m$ or $n$ is zero (but not both).

We are interested in finding the generating function for the set $A_{K, 2 i}(m, n) . B_{K, j}(m, n)$ is thus an auxiliary object whose introduction simplifies considerably the analysis.

Lemma 4 . The sets $A_{K, 2 i}$ and $B_{K, j}$ satisfy the following recurrence relations:

$$
\begin{aligned}
& A_{K, 2 i}(m, n)-A_{K, 2 i-2}(m, n)=B_{K, K-2 i+2}(m-2 i+2, n-i+1), \\
& B_{K, 2 i+1}(m, n)-B_{K, 2 i}(m, n)=A_{K, K-2 i+\epsilon}(m-2 i, n-m) \\
& B_{K, 2 i}(m, n)-B_{K, 2 i-1}(m, n)=A_{K, K-2 i+2-\epsilon}(m-2 i+1, n-m)
\end{aligned}
$$

where $\epsilon$ is related to the parity of $K$ via its decomposition as

$$
K=2 \kappa-\epsilon \quad(\epsilon=0,1) .
$$

Proof: The difference on the left hand side of the recurrence relations selects sets of jagged partitions with a specific boundary term. In particular, $A_{K, 2 i}(m, n)-A_{K, 2 i-2}(m, n)$ gives the number of $K$-restricted jagged partitions of $n$ into $m$ parts containing exactly $i-1$ pairs of 01 at the right. Taking out the tail $01 \cdots 01$, reducing then the length of the partition from $m$ to $m-2(i-1)$ and its weight $n$ by $i-1$, we end up with $K$-restricted jagged partitions which can terminate with a certain number of 1's. These are elements of the set $B_{K, j}(m-2 i+2, n-i+1)$. It remains to fix $j$. The number of 1's in the stripped jagged partitions is constrained by the original $K$-restriction. Before taking out the tail, the number of successive 1 's is at most $K-2(i-1)-1$; this fixes $j$ to be $K-2(i-1)$. We thus get the right hand side of $(i)$. By reversing these operations, we can transform elements of $B_{K, K-2 i+2}(m-2 i+2, n-i+1)$ into those of $A_{K, 2 i}(m, n)-A_{K, 2 i-2}(m, n)$, which shows that the correspondence is one-to-one. This proves $(i)$. 
Consider now the relation $(i i)$. The left hand side is the number of $K$-restricted jagged partitions of $n$ into $m$ parts containing exactly $2 i$ parts equal to 1 at the right end. Subtracting from these jagged partitions the ordinary partition $\left(1^{m}\right)=(1,1,1, \cdots, 1)$ yields new jagged partitions of length $m-2 i$ and weight $n-m$. Since these can have a certain number of pairs of 01 at the end (which is possible if originally we had a sequence of 12 just before the consecutive 1's), we recover elements of $A_{K, 2 i^{\prime}}(m-2 i, n-m)$. It remains to fix $i^{\prime}$. Again, the $K$-restriction puts constraints of the number of allowed pairs 12 in the unstripped jagged partition; it is $\leq(K-2 i+\epsilon-2) / 2$. [Take for instance $K=7$ and $2 i=4$; the lowest-weight jagged partition of length 7 and four 1 's at the end is (2121111), which is compatible with the difference-one condition for parts at distance 6 ; by stripping off $\left(1^{7}\right)$, it is reduced to (101) so that here there is at most one pair of 01 allowed. Take instead $K=8$ and again $2 i=4$; the lowest-weight jagged partition of length 8 is now (22121111), the leftmost 2 being forced by the difference-one condition for parts at distance 7; it is reduced to (1101) so that here there is again at most one pair of 01 allowed. Note that for both these examples, the alternative in-between difference-two condition is not applicable.] Hence $i^{\prime}=(K-2 i+\epsilon) / 2=\kappa-i$. Again the correspondence between sets defined by the two sides of $(i i)$ is one-to-one and this completes the proof of (ii). The proof of $(i i i)$ is similar.

Let us now define the generating functions:

$$
\begin{gathered}
\tilde{A}_{K, 2 i}(z ; q)=\sum_{m, n \geq 0} z^{m} q^{n} A_{K, 2 i}(m, n), \\
\tilde{B}_{K, j}(z ; q)=\sum_{m, n \geq 0} z^{m} q^{n} B_{K, j}(m, n) .
\end{gathered}
$$

In the following, we will generally suppress the explicit $q$ dependence (which will never be modified in our analysis) and write thus $\tilde{A}_{K, 2 i}(z)$ for $\tilde{A}_{K, 2 i}(z ; q)$. The recurrence relations $(i)-($ iii $)$ are now transformed into $q$-difference equations given in the next lemma, whose proof is direct.

Lemma 5. The functions $\tilde{A}_{K, 2 i}(z ; q)$ and $\tilde{B}_{K, j}(z ; q)$ satisfy

$$
\begin{array}{ll}
(i)^{\prime} & \tilde{A}_{K, 2 i}(z)-\tilde{A}_{K, 2 i-2}(z)=\left(z^{2} q\right)^{i-1} \tilde{B}_{K, K-2 i+2}(z), \\
(i i)^{\prime} & \tilde{B}_{K, 2 i+1}(z)-\tilde{B}_{K, 2 i}(z)=(z q)^{2 i} \tilde{A}_{K, K-2 i+\epsilon}(z q), \\
(i i i)^{\prime} & \tilde{B}_{K, 2 i}(z)-\tilde{B}_{K, 2 i-1}(z)=(z q)^{2 i-1} \tilde{A}_{K, K-2 i+2-\epsilon}(z q),
\end{array}
$$

with boundary conditions:

$$
\tilde{A}_{K, 2 i}(0 ; q)=\tilde{A}_{K, 2 i}(z ; 0)=\tilde{B}_{K, j}(0 ; q)=\tilde{B}_{K, j}(z ; 0)=1
$$

and

$$
\tilde{A}_{K, 0}(z)=\tilde{B}_{K, 0}(z)=0
$$

Lemma 6. The solution to Eqs (14)-(16) is unique.

Proof: This follows from the uniqueness of the solutions of (10)-(11), which is itself established by a double induction on $n$ and $i$ (cf. sect. 7.3 in [3]). 
The solution to Eqs (14)-(16) is given by the following theorem, whose proof is reported in the next section.

Theorem 7. The solutions to Eqs (14)-(16) are

$$
\begin{aligned}
& \tilde{A}_{K, 2 i}(z)=\sum_{m_{1}, \cdots, m_{\kappa-1}=0}^{\infty} \frac{\left(-z q^{1+\epsilon m_{\kappa-1}}\right)_{\infty} q^{N_{1}^{2}+\cdots+N_{\kappa-1}^{2}+L_{i}} z^{2 N}}{(q)_{m_{1}} \cdots(q)_{m_{\kappa-1}}}, \\
& \tilde{B}_{K, 2 i}(z)=\sum_{m_{1}, \cdots, m_{\kappa-1}=0}^{\infty} \frac{\left(-z q^{1+\epsilon m_{\kappa-1}}\right)_{\infty} q^{N_{1}^{2}+\cdots+N_{\kappa-1}^{2}+L_{i}+N} z^{2 N}}{(q)_{m_{1}} \cdots(q)_{m_{\kappa-1}}},
\end{aligned}
$$

where $N_{j}$ and $L_{j}$ are defined in (3) with $k$ replaced by $\kappa$ and $\tilde{B}_{K, 2 i+1}(z)$ is obtained from these expressions and $(i i i)^{\prime}$.

Fully developed multiple $q$-series are obtained by expanding $\left(-z q^{1+\epsilon m_{\kappa-1}}\right)_{\infty}$ as

$$
\left(-z q^{1+\epsilon m_{\kappa-1}}\right)_{\infty}=\sum_{m_{0}=0}^{\infty} \frac{z^{m_{0}} q^{m_{0}\left(m_{0}+1\right) / 2} q^{\epsilon m_{0} m_{\kappa-1}}}{(q)_{m_{0}}} .
$$

Corollary 8. For $K=2 \kappa$, the solutions to Eq. (14)-(16) reduce to

$$
\begin{aligned}
& \tilde{A}_{K, 2 i}(z ; q)=(-z q)_{\infty} F_{\kappa, i}\left(z^{2} ; q\right), \\
& \tilde{B}_{K, 2 i}(z ; q)=(-z q)_{\infty} F_{\kappa, i}\left(z^{2} q ; q\right),
\end{aligned}
$$

with $F_{\kappa, i}\left(z^{2} ; q\right)$ defined in $(2)$.

Proof: This follows directly from Theorem 7 with $\epsilon=0$. An alternative direct proof, independent of Theorem 7 , is given in section 5. See also [5].

\section{$4 \quad$ Proof of Theorem 7}

The proof of (17) proceeds as follows (and this argument is much inspired by [2]). One first rewrites the formulas (17) under the form

$$
\begin{aligned}
& \tilde{A}_{K, 2 i}(z)=\sum_{n \geq 0} \frac{\left(-z q^{1+\epsilon n}\right)_{\infty} q^{(\kappa-i) n}\left(z^{2} q^{n}\right)^{(\kappa-1) n}}{(q)_{n}} F_{\kappa-1, i}\left(z^{2} q^{2 n}\right), \\
& \tilde{B}_{K, 2 i}(z)=\sum_{n \geq 0} \frac{\left(-z q^{1+\epsilon n}\right)_{\infty} q^{(2 \kappa-i-1) n}\left(z^{2} q^{n}\right)^{(\kappa-1) n}}{(q)_{n}} F_{\kappa-1, i}\left(z^{2} q^{2 n+1}\right)
\end{aligned}
$$

The function $\tilde{B}_{K, 2 i-1}(z)$ is obtained from these expressions by

$$
\tilde{B}_{K, 2 i-1}(z)=\tilde{B}_{K, 2 i}(z)-(z q)^{2 i-1} \tilde{A}_{K, K-2 i+2-\epsilon}(z q) .
$$

The function $F_{\kappa, i}(z)$ is defined in (2) and it satisfies the recurrence relation:

$$
F_{\kappa, i}(z)-F_{\kappa, i-1}(z)=(z q)^{i-1} F_{\kappa, \kappa-i+1}(z q)
$$


with boundary conditions

$$
F_{\kappa, i}(z ; 0)=F_{\kappa, i}(0 ; q)=1 \quad F_{\kappa, 0}(z)=F_{\kappa,-1}(z)=0 .
$$

Note that the vanishing of $F_{\kappa, 0}(z)$ together with the recurrence relation (22) imply that

$$
F_{\kappa, 1}(z)=F_{\kappa, \kappa}(z q) .
$$

The multiple $q$-series (2) is the unique solution of (22) with the specified boundary conditions [2].

We will now show that the expressions (20) satisfy the recurrence relations (14) and the boundary conditions (15) and (16). The latter are immediately verified: the vanishing of $F_{\kappa-1,-1}(z)$ implies that of $\tilde{A}_{K, 0}(z)$ and $\tilde{B}_{K, 0}(z)$, while the precise form of (20) together with the fact that $F_{\kappa, i}(z ; q)$ is equal to 1 if either $z$ or $q$ vanishes ensure the validity of (15).

Let us first verify the relation $(i)^{\prime}$ :

$$
\begin{aligned}
\tilde{A}_{K, 2 i}(z)-\tilde{A}_{K, 2 i-2}(z)=\sum_{n \geq 0} \frac{\left(-z q^{1+\epsilon n}\right)_{\infty} q^{(\kappa-i) n}\left(z^{2} q^{n}\right)^{(\kappa-1) n}}{(q)_{n}} & \\
& \times\left[F_{\kappa-1, i}\left(z^{2} q^{2 n}\right)-q^{n} F_{\kappa-1, i-1}\left(z^{2} q^{2 n}\right)\right] .
\end{aligned}
$$

In the first step, we reorganize the square bracket as

$$
F_{\kappa-1, i}\left(z^{2} q^{2 n}\right)-F_{\kappa-1, i-1}\left(z^{2} q^{2 n}\right)+\left(1-q^{n}\right) F_{\kappa-1, i-1}\left(z^{2} q^{2 n}\right)
$$

and then replace the first two terms by $\left(z^{2} q^{2 n+1}\right)^{i-1} F_{\kappa-1, \kappa-i}\left(z^{2} q^{2 n+1}\right)$ using (22). That leads to

$$
\tilde{A}_{K, 2 i}(z)-\tilde{A}_{K, 2 i-2}(z)=R_{1}+R_{2}
$$

with

$$
R_{1}=\left(z^{2} q\right)^{i-1} \sum_{n \geq 0} \frac{\left(-z q^{1+\epsilon n}\right)_{\infty} q^{(\kappa+i-2) n}\left(z^{2} q^{n}\right)^{(\kappa-1) n}}{(q)_{n}} F_{\kappa-1, \kappa-i}\left(z^{2} q^{2 n+1}\right)
$$

and

$$
R_{2}=\sum_{n \geq 1} \frac{\left(-z q^{1+\epsilon n}\right)_{\infty} q^{(\kappa-i) n}\left(z^{2} q^{n}\right)^{(\kappa-1) n}}{(q)_{n-1}} F_{\kappa-1, i-1}\left(z^{2} q^{2 n}\right)
$$

(note that the summation in $R_{2}$ starts at $n=1$ and $(q)_{n}$ in the denominator has been changed to $(q)_{n-1}$ to cancel the $\left(1-q^{n}\right)$ in numerator.) Let us leave $R_{2}$ for the moment and manipulate $R_{1}$. First write

$$
F_{\kappa-1, \kappa-i}\left(z^{2} q^{2 n+1}\right)=F_{\kappa-1, \kappa-i+1}\left(z^{2} q^{2 n+1}\right)-\left[F_{\kappa-1, \kappa-i+1}\left(z^{2} q^{2 n+1}\right)-F_{\kappa-1, \kappa-i}\left(z^{2} q^{2 n+1}\right)\right]
$$

and use again $(22)$ to replace the last two terms by $-\left(z^{2} q^{2 n+2}\right)^{\kappa-i} F_{\kappa-1, i-1}\left(z^{2} q^{2 n+2}\right)$. We have thus decomposed $R_{1}$ in two pieces:

$$
R_{1}=S_{1}+S_{2}
$$


with

$$
\begin{aligned}
S_{1} & =\left(z^{2} q\right)^{i-1} \sum_{n \geq 0} \frac{\left(-z q^{1+\epsilon n}\right)_{\infty} q^{(\kappa+i-2) n}\left(z^{2} q^{n}\right)^{(\kappa-1) n}}{(q)_{n}} F_{\kappa-1, \kappa-i+1}\left(z^{2} q^{2 n+1}\right) \\
& =\left(z^{2} q\right)^{i-1} \tilde{B}_{K, K-2 i+2+\epsilon}(z)
\end{aligned}
$$

(to fix the second subindex of $B$ observe that $K-2 i+2+\epsilon=2(\kappa-i+1)$ ) and

$$
S_{2}=-\left(z^{2} q\right)^{i-1} \sum_{n \geq 0} \frac{\left(-z q^{1+\epsilon n}\right)_{\infty}\left(z^{2} q^{n+2}\right)^{(\kappa-1) n+(\kappa-i)}}{(q)_{n}} F_{\kappa-1, i-1}\left(z^{2} q^{2 n+2}\right) .
$$

Summing up our results at this point, we have

$$
\tilde{A}_{K, 2 i}(z)-\tilde{A}_{K, 2 i-2}(z)=\left(z^{2} q\right)^{i-1} \tilde{B}_{K, K-2 i+2+\epsilon}(z)+S_{2}+R_{2} .
$$

Let us now come back to $R_{2}$. We first shuffle the index $n$ to start its summation at zero:

$$
R_{2}=\left(z^{2} q\right)^{i-1} \sum_{n \geq 0} \frac{\left(-z q^{1+\epsilon(n+1)}\right)_{\infty}\left(z^{2} q^{n+2}\right)^{(\kappa-1) n+(\kappa-i)}}{(q)_{n}} F_{\kappa-1, i-1}\left(z^{2} q^{2 n+2}\right) .
$$

From now on, we will use the following compact notation:

$$
\left(-z q^{1+\epsilon n}\right)_{\infty} f_{m}=\sum_{m=0}^{\infty} \frac{z^{m} q^{m(m+1) / 2} q^{\epsilon m n}}{(q)_{m}} f_{m}
$$

i.e., we understand that $\left(-z q^{1+\epsilon n}\right)_{\infty}$ is defined by its sum expression over $m$ so that it makes sense to insert at its right a term that depends upon $m$. With that notation, shifting $n$ by one unit yields:

$$
\left(-z q^{1+\epsilon(n+1)}\right)_{\infty}=\left(-z q^{1+\epsilon n}\right)_{\infty} q^{\epsilon m}
$$

$R_{2}$ reads thus

$$
R_{2}=\left(z^{2} q\right)^{i-1} \sum_{n \geq 0} \frac{\left(-z q^{1+\epsilon n}\right)_{\infty} q^{\epsilon m}\left(z^{2} q^{n+2}\right)^{(\kappa-1) n+(\kappa-i)}}{(q)_{n}} F_{\kappa-1, i-1}\left(z^{2} q^{2 n+2}\right) .
$$

By comparing this expression with that of $S_{2}$, we find that the summand in $R_{2}$ and $S_{2}$ are exactly the same except for the sign and an extra factor $q^{\epsilon m}$ in $R_{2}$ :

$$
S_{2}+R_{2}=-\left(z^{2} q\right)^{i-1} \sum_{n \geq 0} \frac{\left(-z q^{1+\epsilon n}\right)_{\infty}\left(z^{2} q^{n+2}\right)^{(\kappa-1) n+(\kappa-i)}}{(q)_{n}}\left(1-q^{\epsilon m}\right) F_{\kappa-1, i-1}\left(z^{2} q^{2 n+2}\right)
$$

A simple observation here is that $1-q^{\epsilon m}$ vanishes if $\epsilon=0$. Since $\epsilon$ can take only the values 0 or 1 , we can thus write

$$
\left(1-q^{\epsilon m}\right)=\epsilon\left(1-q^{m}\right)
$$


$S_{2}+R_{2}$ is thus proportional to $\epsilon$ and we can evaluate the proportionality factor at $\epsilon=1$. It is simple to check that

$$
\left(-z q^{1+\epsilon n}\right)_{\infty}\left(1-q^{m}\right)=z q\left(-z q^{1+\epsilon n}\right)_{\infty} q^{m+\epsilon n} .
$$

To be explicit: this is obtained from $\left(1-q^{m}\right) /(q)_{m}=1 /(q)_{m-1}$ and by shuffling the $m$ index in the $m$-summation. Similarly, replacing $z \rightarrow z q$ in $\left(-z q^{1+\epsilon n}\right)_{\infty}$ leads to

$$
\left(-z q^{2+\epsilon n}\right)_{\infty}=\left(-z q^{1+\epsilon n}\right)_{\infty} q^{m} .
$$

The comparison of the last two results gives

$$
\left(-z q^{1+\epsilon n}\right)_{\infty}\left(1-q^{m}\right)=z q\left(-z q^{2+\epsilon n}\right)_{\infty} q^{\epsilon n} .
$$

Substituting this into the expression of $S_{2}+R_{2}$ (and setting $\epsilon=1$ when it appears in an exponent) leads to

$$
S_{2}+R_{2}=-\epsilon\left(z^{2} q\right)^{i-1}(z q)^{2(\kappa-i)+1} \tilde{A}_{K, 2 i-2}(z q) .
$$

Note that we can replace $2 \kappa$ by $K+1$ (since $\epsilon=1$ ) in the exponent of $z q$.

Collecting all our results, we have

$$
\begin{aligned}
\tilde{A}_{K, 2 i}(z)-\tilde{A}_{K, 2 i-2}(z) & =\left(z^{2} q\right)^{i-1}\left[\tilde{B}_{K, K-2 i+2+\epsilon}(z)-\epsilon(z q)^{K-2 i+2} \tilde{A}_{K, 2 i-2}(z q)\right] \\
& =\left(z^{2} q\right)^{i-1} \tilde{B}_{K, K-2 i+2}(z)
\end{aligned}
$$

since $\tilde{B}_{K, K-2 i+2+\epsilon}$ is equal to $\tilde{B}_{K, K-2 i+2}$ if $\epsilon=0$ or is given by (21) if $\epsilon=1$. We have thus completed the verification of $(i)^{\prime}$.

We now turn to the relation $(i i)^{\prime}$. Note that the left hand side is not expressible directly in terms of a summand times a difference of $F$-functions due to the presence of $\tilde{B}_{K, 2 i+1}$. The first step amounts to reexpressing it in terms of $\tilde{B}_{K, 2 i+2}$ :

$$
\tilde{B}_{K, 2 i+1}(z)-\tilde{B}_{K, 2 i}(z)=\tilde{B}_{K, 2 i+2}(z)-\tilde{B}_{K, 2 i}(z)-(z q)^{2 i+1} \tilde{A}_{K, K-2 i-\epsilon}(z q) .
$$

Let us first concentrate on the difference between the two $\tilde{B}$ factors:

$$
\begin{aligned}
& \tilde{B}_{K, 2 i+2}(z)-\tilde{B}_{K, 2 i}(z)= \sum_{n \geq 0} \frac{\left(-z q^{1+\epsilon n}\right)_{\infty} q^{(2 \kappa-i-2) n}\left(z^{2} q^{n}\right)^{(\kappa-1) n}}{(q)_{n}} \\
& \quad \times\left[F_{\kappa-1, i+1}\left(z^{2} q^{2 n+1}\right)-q^{n} F_{\kappa-1, i}\left(z^{2} q^{2 n+1}\right)\right] .
\end{aligned}
$$

Again, we decompose the term in square bracket as follows

$$
\left[F_{\kappa-1, i+1}\left(z^{2} q^{2 n+1}\right)-F_{\kappa-1, i}\left(z^{2} q^{2 n+1}\right)\right]+\left(1-q^{n}\right) F_{\kappa-1, i}\left(z^{2} q^{2 n+1}\right)
$$

substitute this into the previous equation and write the corresponding two terms as $R_{1}^{\prime}+$ $R_{2}^{\prime}$. With the identity (37), $R_{2}^{\prime}$ takes the form

$$
R_{2}^{\prime}=z^{2 \kappa-2} q^{3 \kappa-i-3} \sum_{n \geq 0} \frac{\left(-z q^{1+\epsilon n}\right)_{\infty} q^{\epsilon m} q^{(4 \kappa-i-4) n}\left(z^{2} q^{n}\right)^{(\kappa-1) n}}{(q)_{n}} F_{\kappa-1, i}\left(z^{2} q^{2 n+3}\right)
$$


On the other hand, $R_{1}^{\prime}$, using $(22)$, reads

$$
R_{1}^{\prime}=(z q)^{2 i} \sum_{n \geq 0} \frac{\left(-z q^{1+\epsilon n}\right)_{\infty} q^{(2 \kappa+i-2) n}\left(z^{2} q^{n}\right)^{(\kappa-1) n}}{(q)_{n}} F_{\kappa-1, \kappa-i-1}\left(z^{2} q^{2 n+2}\right) .
$$

In order to demonstrate $(i i)^{\prime}$, the target is to recover, within the expression of $\tilde{B}_{K, 2 i+1}(z)-$ $\tilde{B}_{K, 2 i}(z)$, that of $(z q)^{2 i} \tilde{A}_{K, K-2 i+\epsilon}(z q)$, which reads (using (42))

$$
(z q)^{2 i} \tilde{A}_{K, K-2 i+\epsilon}(z q)=(z q)^{2 i} \sum_{n \geq 0} \frac{\left(-z q^{1+\epsilon n}\right)_{\infty} q^{m} q^{(2 \kappa+i-2) n}\left(z^{2} q^{n}\right)^{(\kappa-1) n}}{(q)_{n}} F_{\kappa-1, \kappa-i}\left(z^{2} q^{2 n+2}\right) .
$$

Apart from the factor of $q^{m}$ and the value of the second index of the function $F$, the last two expressions are identical. This indicates the way we should manipulate $R_{1}^{\prime}$. First write

$$
F_{\kappa-1, \kappa-i-1}\left(z^{2} q^{2 n+2}\right)=F_{\kappa-1, \kappa-i}\left(z^{2} q^{2 n+2}\right)-\left[F_{\kappa-1, \kappa-i}\left(z^{2} q^{2 n+2}\right)-F_{\kappa-1, \kappa-i-1}\left(z^{2} q^{2 n+2}\right)\right] .
$$

This decomposes $R_{1}^{\prime}$ in two pieces $S_{1}^{\prime}+S_{2}^{\prime}$ with

$$
S_{1}^{\prime}=(z q)^{2 i} \sum_{n \geq 0} \frac{\left(-z q^{1+\epsilon n}\right)_{\infty} q^{(2 \kappa+i-2) n}\left(z^{2} q^{n}\right)^{(\kappa-1) n}}{(q)_{n}} F_{\kappa-1, \kappa-i}\left(z^{2} q^{2 n+2}\right)
$$

and (using again (22))

$$
S_{2}^{\prime}=-z^{2 \kappa-2} q^{3 \kappa-i-3} \sum_{n \geq 0} \frac{\left(-z q^{1+\epsilon n}\right)_{\infty} q^{(4 \kappa-i-4) n}\left(z^{2} q^{n}\right)^{(\kappa-1) n}}{(q)_{n}} F_{\kappa-1, i}\left(z^{2} q^{2 n+3}\right) .
$$

In $S_{1}^{\prime}$, we then insert a factor $q^{m}$ as follows: $1=q^{m}+\left(1-q^{m}\right)$ and write the resulting two contributions as

$$
S_{1}^{\prime}=(z q)^{2 i} \tilde{A}_{K, K-2 i+\epsilon}(z q)+T_{2}^{\prime}
$$

and (with (41)):

$$
T_{2}^{\prime}=(z q)^{2 i+1} \sum_{n \geq 0} \frac{\left(-z q^{1+\epsilon n}\right)_{\infty} q^{\epsilon n+m} q^{(2 \kappa+i-2) n}\left(z^{2} q^{n}\right)^{(\kappa-1) n}}{(q)_{n}} F_{\kappa-1, \kappa-i}\left(z^{2} q^{2 n+2}\right) .
$$

Collecting the results of this paragraph, we see that to complete the proof of $(i i)^{\prime}$ we only have to show that

$$
R_{2}^{\prime}+S_{2}^{\prime}+T_{2}^{\prime}-(z q)^{2 i+1} \tilde{A}_{K, K-2 i-\epsilon}(z q)=0 .
$$

By comparing $R_{2}^{\prime}$ and $S_{2}^{\prime}$, we notice that their summands are identical, up to the sign and to an extra $q^{\epsilon m}$ in $R_{2}^{\prime} . R_{2}^{\prime}+S_{2}^{\prime}$ contains thus the factor $\left(1-q^{\epsilon m}\right)$ which can be handled as previously (cf. eqs (40) and (41)). The result is

$$
R_{2}^{\prime}+S_{2}^{\prime}=-\epsilon z^{2 \kappa-1} q^{3 \kappa-i-2} \sum_{n \geq 0} \frac{\left(-z q^{1+\epsilon n}\right)_{\infty} q^{m} q^{(4 \kappa-i-3) n}\left(z^{2} q^{n}\right)^{(\kappa-1) n}}{(q)_{n}} F_{\kappa-1, i}\left(z^{2} q^{2 n+3}\right)
$$


Combining next $T_{2}^{\prime}$ with $-(z q)^{2 i+1} \tilde{A}_{K, K-2 i-\epsilon}(z q)$ leads to

$$
\begin{aligned}
T_{2}^{\prime}-(z q)^{2 i+1} \tilde{A}_{K, K-2 i-\epsilon}(z q)= & (z q)^{2 i+1} \sum_{n \geq 0} \frac{\left(-z q^{1+\epsilon n}\right)_{\infty} q^{m} q^{(2 \kappa+i-2+\epsilon) n}\left(z^{2} q^{n}\right)^{(\kappa-1) n}}{(q)_{n}} \\
& \times\left[F_{\kappa-1, \kappa-i}\left(z^{2} q^{2 n+2}\right)-F_{\kappa-1, \kappa-i-\epsilon}\left(z^{2} q^{2 n+2}\right)\right] .
\end{aligned}
$$

Using (22) in a slightly modified form, i.e., as

$$
F_{\kappa-1, \kappa-i}\left(z^{2} q^{2 n+2}\right)-F_{\kappa-1, \kappa-i-\epsilon}\left(z^{2} q^{2 n+2}\right)=\epsilon\left(z^{2} q^{2 n+3}\right)^{(\kappa-i-1)} F_{\kappa-1, i}\left(z^{2} q^{2 n+3}\right),
$$

we are led to

$$
T_{2}^{\prime}-(z q)^{2 i+1} \tilde{A}_{K, K-2 i-\epsilon}(z q)=-\left(R_{2}^{\prime}+S_{2}^{\prime}\right),
$$

which demonstrates (57) and thus $(i i)^{\prime}$.

Finally, with $\tilde{B}_{K, 2 i-1}(z)$ defined by $(21)$, relation $(i i i)^{\prime}$ is an identity. We have thus completed the proof of the relations (20) or equivalently of Theorem 7 .

\section{Two simple applications to partition counting}

By adding the staircase $(m-1, m-2, \cdots, 1,0)$ to the sequence $\left(n_{1}, \cdots n_{m}\right)$, we transform it into an ordinary partition. With $\lambda_{j}=n_{j}+m-j$, the weakly decreasing conditions (5) become

$$
\lambda_{j} \geq \lambda_{j+1} \quad \text { and } \quad \lambda_{j} \geq \lambda_{j+2}+2,
$$

while the $K$-restrictions (6) take the form

$$
\lambda_{j} \geq \lambda_{j+K-1}+K \quad \text { or } \quad \lambda_{j}=\lambda_{j+1}=\lambda_{j+K-2}+K-1=\lambda_{j+K-1}+K-1 .
$$

To transform a generating function for $K$-restricted jagged partitions to one for partitions subject to (62) and (63), we simply need to replace $z^{N}$ by $z^{N} q^{N(N-1) / 2}$. Two limiting cases of our general result are of interest.

Corollary 9. The number of partitions satisfying $\lambda_{j} \geq \lambda_{j+2}+2$ is given by

$$
\lim _{\kappa \rightarrow \infty} \sum_{m, n \geq 0} A_{2 \kappa, 2 \kappa}(m, n) z^{m} q^{m(m-1) / 2+n}=\sum_{m_{0}, m_{1} \geq 0} \frac{q^{\left(m_{0}+m_{1}\right)^{2}+m_{1}^{2} z^{m_{0}+2 m_{1}}}}{(q)_{m_{0}}(q)_{m_{1}}}=F_{3,3}(z) .
$$

Proof: In the limit $\kappa \rightarrow \infty$, the restrictions can be disregarded and we are left with unrestricted jagged partitions, which, by Corollary 8, satisfy

$$
\lim _{\kappa \rightarrow \infty} \mathcal{A}_{2 \kappa, 2 \kappa}(z)=(-z q)_{\infty} \lim _{\kappa \rightarrow \infty} F_{\kappa, \kappa}\left(z^{2}\right)=\frac{(-z q)_{\infty}}{\left(z^{2} q\right)_{\infty}}
$$

The second equality follows from Theorem 2 of [2]. By expanding $(-z q)_{\infty} /\left(z^{2} q\right)_{\infty}$ and replacing $z^{N}$ by $z^{N} q^{N(N-1) / 2}$, we recover the first equality of (64), which is seen to be 
equivalent to $F_{3,3}(z)$, as it should (since we simply recover a special case of [2] quoted in the introduction).

Corollary 10. The number of partitions satisfying $\lambda_{j} \geq \lambda_{j+2}+3$ is given by

$$
\sum_{m, n \geq 0} A_{3,4}(m, n) z^{m} q^{m(m-1) / 2+n}=\sum_{m_{0}, m_{1} \geq 0} \frac{q^{m_{0}^{2}+3 m_{1}\left(m_{0}+m_{1}-1 / 3\right)} z^{m_{0}+2 m_{1}}}{(q)_{m_{0}}(q)_{m_{1}}} .
$$

Proof: For $K=3$, all the restrictions on the partitions defined by (62) and (63) reduce to

$$
\lambda_{j} \geq \lambda_{j+2}+3
$$

We can apply (17) to the counting of such partitions by considering $\mathcal{A}_{3,4}(z, q)$ (i.e., setting $i=2$ to take into account all boundary conditions). Replacing again $z^{N}$ by $z^{N} q^{N(N-1) / 2}$, leads to (66).

This provides a quite simple derivation of a specialization of the generating function of partitions $\left(\lambda_{1}, \cdots, \lambda_{m}\right)$ satisfying $\lambda_{i} \geq \lambda_{i+k-1}+3$ obtained in [11] (cf. their Eq 6). (See also Theorem 9.9 of [12] for the generating functions of the restricted partitions for this generic case (all $k$ ). Our result is also a specialization of the one presented in Theorem 5.14 of [10] pertaining to the case $\lambda_{i} \geq \lambda_{i+2}+\ell$.)

\section{Product form of the specialized generating func- tion}

Let us return to the general multiple $\operatorname{sum} \tilde{A}_{K, 2 i}(z)=\tilde{A}_{K, 2 i}(z ; q)$. For $z=1$, it can be regarded as the sum-side of a generalized version of the Rogers-Ramanujan identities. In this section, we display the corresponding product form together with its combinatorial interpretation.

Theorem 11. The product form of $\tilde{A}_{K, 2 i}(1 ; q)$, with $K=2 \kappa-\epsilon$ and $1 \leq i \leq \kappa$ reads

$$
\begin{aligned}
\tilde{A}_{K, 2 i}(1 ; q) & =\prod_{n=1}^{\infty}\left(1+q^{n}\right) \prod_{n \neq 0, \pm i \bmod (K+1)}^{\infty}\left(1-q^{n}\right)^{-1} \quad(\epsilon=0,1, ; i<(K+1) / 2) \\
& =\prod_{n \neq 0 \bmod \kappa}^{\infty}\left[\left(1+q^{n}\right)\left(1-q^{n}\right)^{-1}\right] \quad(\epsilon=1, i=\kappa) .
\end{aligned}
$$

Proof: Using the simple identity $\left(-q^{1+\epsilon m_{\kappa-1}}\right)_{\infty}=(-q)_{\infty} /(-q)_{\epsilon m_{\kappa-1}}$, we can rewrite $\tilde{A}_{K, 2 i}$, given by (17), as

$$
\tilde{A}_{K, 2 i}(1 ; q)=(-q)_{\infty} \sum_{m_{1}, \cdots, m_{\kappa-1}=0}^{\infty} \frac{q^{N_{1}^{2}+\cdots+N_{\kappa-1}^{2}+L_{i}}}{(q)_{m_{1}} \cdots(q)_{m_{\kappa-2}}\left(q^{1+\epsilon} ; q^{1+\epsilon}\right)_{m_{\kappa-1}}} .
$$


Up to the prefactor $(-q)_{\infty}$, the multiple sum is now in a form equivalent to one used in [6]. The result $(68)$ for $i<(K+1) / 2$ follows directly from Theorem 1 of [6]. It only remains to consider the case where $\epsilon=1$ and $i=(K+1) / 2=\kappa$. But this is implicitly treated in Lemma 1 of [6], which leads immediately to the second line of (68). (In that case, the restriction $n \neq 0, \pm \kappa \bmod 2 \kappa$ reduces to $n \neq 0 \bmod \kappa$.)

Manifestly, in all cases but $\epsilon=1, i=\kappa$, the factor $(-q)_{\infty}$ can be dropped from both sides of (68) (cf. (69) for the left hand side). By doing so, we recover the Andrews-Gordon identities $(\epsilon=0)$ [1] and the Bressoud identities $(\epsilon=1)$ [6]. For $\epsilon=1, i=\kappa,(68)$ appears to be a new identity.

Note that for $\epsilon=1, i<\kappa$, we have the following expression:

$$
\tilde{A}_{2 \kappa-1,2 i}(1 ; q)=\frac{(-q)_{\infty}}{(q)_{\infty}}\left(q^{i}, q^{2 \kappa-i}, q^{2 \kappa} ; q^{2 \kappa}\right)_{\infty}
$$

For $i=1$, this is equal to $F_{\kappa, 1}(-1 / q ; 1 ; q)$ (cf. Lem. 2.6 of [16]), a specialization of the function $F_{\kappa, i}(a ; z ; q)$ of Andrews $[2,3]\left(F_{\kappa, i}(z ; q)\right.$ in $(2)$ being its $a=0$ version). For $i=\kappa$, we have

$$
\tilde{A}_{2 \kappa-1,2 \kappa}(1, q)=\frac{(-q)_{\infty}}{(q)_{\infty}} \frac{\left(q^{\kappa} ; q^{\kappa}\right)_{\infty}}{\left(-q^{\kappa} ; q^{\kappa}\right)_{\infty}}=F_{\kappa, \kappa}(-1 ; 1 ; q)
$$

where the last identity is proved in [16], Lem. 2.5. For $i=2, K=3$, this is the product side of Lebesgue's identity (cf. [3] Cor. 2.7 with $a=1$ ).

The combinatorial interpretation of the Rogers-Ramanujan-type identities (68) (with $\tilde{A}_{K, 2 i}$ given by (17)) relies on the description of jagged partitions as overpartitions. Recall that an overpartition is a partition in which the first occurence of a number may be overlined [9]. An overpartition is thus equivalent to a pair $(\bar{\alpha}, \beta)$ of partitions with the constraint that the parts of $\bar{\alpha}$ are distinct (i.e., they are the overlined parts). There is a natural bijection between overpartitions and jagged partitions, obtained as follows [17]. Replace adjacent integers $(n, n+1)$ within the jagged partition by $2 n+1$ and similarly replace adjacent integers $(n, n)$ by $2 n$. The numbers thus obtained form the parts of $\beta$. The remaining entries of the jagged partitions are necessarily non-zero and distinct integers; they build up $\bar{\alpha}$. On the other hand, given an overpartition $(\bar{\alpha}, \beta)$, one first decomposes all entries of $\beta$ according to their parity, either as $2 n=(n, n)$ or $2 n+1=(n, n+1)$ and uses the resulting (adjacent) parts together with those of $\bar{\alpha}$, to construct a jagged partition according to the restrictions (5). This is unique and this demonstrates the bijective character of the correspondence. (Observe that the equivalence between the set of jagged partitions of weight $n$ and pairs of partitions $(\bar{\alpha}, \beta)$ whose weights add up to $n$, is a direct consequence of the generating function (65)). A similar bijection between jagged partitions and overpartitions has also been obtained in [18].

The above bijection and Theorem 11 lead directly to the following.

Corollary 12. The number $A_{K, 2 i}(n)$ (with $1 \leq i \leq[(K+1) / 2]$ and $K=2 \kappa-\epsilon$ ) of jagged partitions of weight $n$ satisfying the restrictions (6) and containing at most $i-1$ pairs 01 is equal to the number of overpartitions $(\bar{\alpha}, \beta)$ of combined weight $n$ where parts 
of $\beta$ are not equal to $0, \pm i \bmod K+1$ if $1 \leq i<(K+1) / 2$ or where no part of $\bar{\alpha}$ and $\beta$ is equal to $0 \bmod \kappa$ if $\epsilon=1$ and $i=\kappa$.

A completely different combinatorial interpretation of the identity (68) (without the $(-q)_{\infty}$ factor) is given in $[1,6]$ (except for the case $\epsilon=1$ and $i=\kappa$ which is not covered in [6]). We stress that by including a $(-q)_{\infty}$ factor, we end up with a new combinatorial description of these previously known identities. Note also that the sum-side does not seem to have a natural interpretation in terms of overpartitions with a difference condition between parts at distance $K-1$.

\section{Complementary remarks}

Before concluding, we would like to present some clarifying remarks. The first one concerns the relationship between the $K$-restrictions (6) and the recurrence relations (11). A basic observation is that they have a dual role: the restriction conditions specify the allowed jagged partitions, while the recurrence relations are controlled by the excluded jagged partitions. Hence, if at first sight it might not seem natural to have a restriction formulated in terms of an 'or'-type condition, it is clear that the introduction of an alternative allows for more jagged partitions than with a single restriction. And this implies that there are less excluded jagged partitions, meaning, in turn, that the recurrence relations are simplified. In other words, if the restriction was formulated solely as a difference-one condition for parts at distance $K-1$, that would result in a system of recurrence relations more complicated than (11) and unlikely to be solvable in closed form.

To make the duality more explicit, observe that the $K$-restrictions (6) are equivalent to excluding all jagged partitions containing a subsequence $\left(n_{j}, \cdots, n_{j+K-1}\right)$ of either one of the following form:

$$
(\underbrace{p, \cdots, p}_{K-2 \ell}, \underbrace{p-1, p, \cdots, p-1, p}_{2 \ell}) \quad \text { or } \quad(\underbrace{p, p+1, \cdots, p, p+1}_{2 \ell}, \underbrace{p, \cdots, p}_{K-2 \ell})
$$

with $0 \leq \ell \leq[K / 2]$. When viewed from this angle, the naturalness of the condition (6) reveals itself: it amounts to excluding precisely one subsequence of length $K$ for each value of the weight $n=\sum_{r=j}^{K-j+1} n_{r}(n \geq[(K+1) / 2])$. But this pattern of excluded subsequences pops up directly from the recurrence relations. The condition $(i)$ indicates that we need to exclude all sequences whose tail is of the form

$$
(\cdots, \underbrace{1, \cdots, 1}_{K-2 \ell}, \underbrace{0,1, \cdots, 0,1}_{2 \ell})
$$

(with $\ell=i-1$ ), while the conditions (ii) and (iii) amounts to eliminating all sequences with the following tail:

$$
(\cdots, \underbrace{1,2, \cdots, 1,2}_{2 \ell}, \underbrace{1, \cdots, 1}_{K-2 \ell})
$$

THE ELECTRONiC JOURNAL OF COMBINATORICS 12 (2005), \#R12 
(with $\ell=\kappa-i$ or $\kappa-i+1-\epsilon$ respectively). Since the restriction conditions are invariant under a shift of all the parts $n_{j}$ by the same integer $p$ (i.e., by adding $\left(p^{m}\right)$ to the jagged partition), the exclusion condition must share this invariance property; this lifts the tailexclusions (73) and (74) to the general ones (72).

Next, since our proof of Theorem 7 is not constructive and based on the judicious ansatz (17), it is fair to present some rationale underlying this ansatz. At first, we already knew from [5] that for $K=2 \kappa$,

$$
\tilde{A}_{K, 2 i}(z)=(-z q)_{\infty} F_{\kappa, i}\left(z^{2}\right) .
$$

Here is a very quick proof, independent of Theorem 7. Set

$$
\tilde{A}_{K, 2 i}(z)=f(z) F_{\kappa, i}\left(z^{2}\right)
$$

and substitute this into $(i)^{\prime}$, using (22); this leads to

$$
\tilde{B}_{K, 2 i}(z)=f(z) F_{\kappa, i}\left(z^{2} q\right) \text {. }
$$

Then from $(i i i)^{\prime}$ we get

$$
\tilde{B}_{K, 2 i-1}(z)=f(z ; q) F_{\kappa, i}\left(z^{2} q\right)-z^{2 i-1} q^{2 i-1} f(z q) F_{\kappa, \kappa-i+1}\left(z^{2} q^{2}\right) .
$$

The substitution of these expressions into $(i i)^{\prime}$ yields then

$$
f(z ; q)=(1+z q) f(z q) \quad \Rightarrow \quad f(z)=(-z q)_{\infty} .
$$

In that case, the $m_{0}$ mode defined by the sum expression of $(-z q)_{\infty}$ is thus independent of the $m_{j}$ ones of $F_{\kappa, i}$. Given that in the large- $K$ limit, the parity of $K$ should not matter anymore, we should recover the above simple result even for $K$ odd, as $K \rightarrow \infty$. This means that if the $m_{0}$ mode is coupled to some other modes $m_{j}$ when $K$ is odd, this coupling should disappear as $K \rightarrow \infty$. In the multiple-sum expression (1) of $F_{\kappa, i}$, we see

that there are terms like $q^{j m_{j}^{2}}$, so that in the large $K$ (or $\kappa$ ) limit, the only contributing values of the modes $m_{j}$ with $j$ of the order of $\kappa$ are $m_{j}=0$ (with the usual assumption that $q<1$ ). From these considerations, we thus knew that $m_{0}$ could couple only with those modes $m_{j}$ with $j$ of the order of $\kappa$. The natural guess is to look for a single coupling with the mode with largest subindex, $m_{\kappa-1}$. This is also a very natural hypothesis if we expect an iterative formula like (20) to exist (where the iteration is on $\kappa$ ) in which the dependence upon the modes $m_{j}, 1 \leq j \leq \kappa-2$ is factored out.

\section{Conclusion}

We have presented a rather interesting extension of the generating function counting partitions whose parts separated by distance $k-1$ differ by at least 2 , by enumerating novel types of partitions (dubbed 'jagged') subject to a new type of restriction. That 
the rather complicated restriction considered here (dictated, as already pointed out, by a physical problem) leads to a set of $q$-difference equations solvable by functions so similar to the original Andrews' multiple sums is certainly quite remarkable.

The jagged partitions that have been considered in the present work are characterized by the nature of their lowest-weight sequence which is $(\cdots 010101)$. It is clear that there is a whole hierarchy of jagged-type partitions generalizing those considered here. The simplest generalizations are characterized by the following lowest-weight sequences : $(\cdots 001001),(\cdots 020202)$ and $(\cdots 012012)$. The generating functions enumerating those specific generalized jagged partitions are presented in [13]. However, we have not found the proper way of imposing restrictions on these jagged partitions that would lead to tracktable generating functions. For that quest, we had no guide from physical models.

As already stressed, the main interest of this work is probably rooted in the new light it sheds on the following well-known generalization of the Ramanujan-Rogers identities (with $K=2 \kappa-\epsilon$ and $i<(K+1) / 2$ ):

$$
\sum_{m_{1}, \cdots, m_{\kappa-1}=0}^{\infty} \frac{q^{N_{1}^{2}+\cdots+N_{\kappa-1}^{2}+L_{i}}}{(q)_{m_{1}} \cdots(q)_{m_{\kappa-2}}\left(q^{1+\epsilon} ; q^{1+\epsilon}\right)_{m_{\kappa-1}}}=\prod_{n \neq 0, \pm i \bmod (K+1)}^{\infty}\left(1-q^{n}\right)^{-1}
$$

The combinatorial interpretation of this identity is presented in [6]. Our new interpretation, in terms of jagged partitions, does not apply directly to the above identity, however. It merely provides an interpretation of a dressed version of it, that is, the identity that results from multiplying both sides by $(-q)_{\infty}$ (cf. Corollary 12). In addition, it covers one case that is not considered in [6] $(\epsilon=1$ and $i=\kappa)$.

Note finally that the sum side of (80) has been reinterpreted in terms of lattice paths in [7] (building on the works [8] and [4]). This poses the natural problem: what type of lattice paths are enumerated by the multiple sum $(80)$ times $(-q)_{\infty}$ ?

\section{ACKNOWLEDGMENTS}

We thank J. Lovejoy for communicating to us the bijection between overpartitions and jagged partitions and also for pointing out references [6] and [18]. We also thank the referee for drawing our attention to lattice paths.

\section{References}

[1] G.E. Andrews, An analytic generalization of the Rogers-Ramanujan identities for odd moduli, Proc. Nat. Acad. Sci. USA 71 (1974) 4082-4085.

[2] G.E. Andrews, Multiple q-series, Houston J. Math. 7 (1981) 11-22.

[3] G.E. Andrews, The theory of partitions, Cambridge Univ. Press, Cambridge, UK, (1984). 
[4] G.E. Andrews and D. Bressoud, On the Burge correspondence between partitions and binary words, Rocky Mtn J. Math. 15 (1985) 225-233.

[5] L. Bégin, J.-F. Fortin, P. Jacob and P. Mathieu, Fermionic characters for graded parafermions, Nucl. Phys B659 (2003) 365-386.

[6] D. Bressoud, An analytic generalization of the Rogers-Ramanujan identities with interpretation, Quart. J. Math. Oxford (2), 31 (1980) 385-399.

[7] D. Bressoud, Lattice paths and Rogers-Ramanujan identities, in Number Theory, Madras 1987, ed. K. Alladi. Lecture Notes in Mathematics 1395 (1987) 140-172.

[8] W.H. Burge, A correspondence between partitions related to generalizations of the Rogers-Ramanujan identities, Discrete Math. 34 (1981) 9-15.

[9] S. Cortel and J. Lovejoy, Overpartitions, Trans. Amer. Math. Soc. 356 (2004), 16231635.

[10] B. Feigin, M. Jimbo, T. Miwa,, Vertex operator algebra arising from the minimal series $M(3, p)$ and monomial basis, math.QA/0012193.

[11] B. Feigin, M. Jimbo, T. Miwa, E. Mukhin and Y. Takeyama, Particle content of the (k,3)-configurations, math.QA/0212348.

[12] B. Feigin, M. Jimbo, T. Miwa, E. Mukhin and Y. Takeyama, Fermionic formulas for (k, 3)-admissible configurations, math.QA/0212347.

[13] J.-F. Fortin, P. Jacob and P. Mathieu, Jagged partitions, to appear in Ramanujan J., math.CO/0310079.

[14] J.-F. Fortin, P. Jacob and P. Mathieu, $S M(2,4 \kappa)$ fermionic characters and restricted jagged partitions, to appear in J. Phys. A: Math. Gen. 38 (2005), hep-th/0406194.

[15] P. Jacob and P. Mathieu, Graded parafermions: standard and quasi-particle bases, Nucl. Phys. B630 (2002) 433-452.

[16] J. Lovejoy, Gordon's theorem for overpartitions, J. Comb. Theory Ser. A 1032003 , no 2, 393-401.

[17] J. Lovejoy, private communication.

[18] K. Mahlburg, The overpartition function modulo small powers of 2, Discrete Math. 286 (2004), 263-267. 